\title{
Mucormycosis in COVID-19 pandemic and its neurovascular spread
}

\author{
Pooja Pal ${ }^{1}$ (D) Bikramjit Singh ${ }^{2}$ (1) $\cdot$ Sumant Singla ${ }^{1} \cdot$ Rupinder Kaur $^{2}$
}

Received: 26 July 2021 / Accepted: 20 September 2021 / Published online: 12 October 2021

(c) The Author(s), under exclusive licence to Springer-Verlag GmbH Germany, part of Springer Nature 2021

\begin{abstract}
Purpose Rhinocerebral mucormycosis is a rapidly progressive angioinvasive fungal infection commonly seen in diabetics. In the COVID-19 pandemic we have witnessed a sudden surge in these cases. We aimed to evaluate the disease presentation, patterns of spread, and any association with the COVID-19 virus.

Methods This prospective study was conducted on mucormycosis patients operated between March and July 2021. The diagnosis was confirmed either on $\mathrm{KOH}$ staining, fungal culture or histopathological examination.

Results Thirty one cases (21 males, 10 females) with a mean age of 53.3 years were included, of which $9(29.1 \%)$ were COVID positive on presentation, 17 (54.8\%) were post-COVID, while 5 (16.1\%) had radiological evidence of COVID sequelae. Most common symptoms were cheek numbness (87.1\%), headache (83.9\%), visual disturbances (77.4\%), and palate involvement (58.1\%). Blackening of turbinates was uncommon (22.6\%). Ethmoid sinus was involved in all patients. Pterygopalatine fossa involvement was present in $77.4 \%$, and was accurately diagnosed on contrast enhanced MRI scan. There were 8 (25.8\%) deaths, while the remaining are discharged or under treatment.

Conclusion An increase in the incidence of mucormycosis in the COVID-19 pandemic is probably due to a compromise in host immunity along with a synergistic effect in thrombotic microangiopathy. Spread of infection to the soft tissues of the infratemporal fossa, orbit or palate occur via neurovascular structures rather than by bone erosion. The pterygopalatine fossa is involved in most individuals.
\end{abstract}

Keywords COVID-19 pandemic $\cdot$ Mucormycosis $\cdot$ Pterygopalatine fossa $\cdot$ Thrombotic microangiopathy

\section{Introduction}

Mucormycosis is a rapidly progressive angioinvasive infection caused by fungi belonging to the Mucorales order. Depending on the site of involvement, it may be classified as rhinocerebral, pulmonary, gastrointestinal, disseminated, or other rare forms [1]. A disease predominantly restricted to individuals with compromised immunity, it is usually seen in patients of diabetes mellitus with or without ketoacidosis, organ transplant recipients, patients with hematological

Bikramjit Singh

drsinghbj@gmail.com

Pooja Pal

drpoojapal@gmail.com

1 Department of Otolaryngology and Head Neck, Sri Guru Ram Das Institute of Health Sciences and Research, Vallah, Amritsar, Punjab, India

2 Department of Surgical Oncology, Government Medical College, Amritsar, Punjab, India malignancies, hepatic or renal failure, and patients on prolonged steroid therapy or chemotherapy [2]. The etiology may also be linked to the site of disease, with rhinocerebral mucormycosis commonly seen in diabetics.

Compared to developed countries, India has a higher prevalence of approximately 0.14 cases of mucormycosis per 1000 population [3]. However, since the onset of the second wave of the COVID-19 pandemic earlier this year, there has been an unprecedented rise in the number of cases of mucormycosis. In the present study, we aimed to look at the disease presentation, patterns of spread, and any correlation with the novel coronavirus.

\section{Materials and methods}

The present prospective study was conducted on patients undergoing surgery for invasive fungal sinusitis at a tertiary care centre in Punjab, India, between Mar 2021 and 15th Jul 2021. Detailed history was taken from the patients 
regarding symptomatology, presence of any comorbidities, and past history of COVID-19 infection. The patients were subjected to a complete ENT evaluation with special focus on examination of the premaxilla, palate and alveolar ridges. Diagnostic nasal endoscopy was done to look for any obvious lesion in the form of blackening or necrosis.

Complete ophthalmologic evaluation was also performed to look for any evidence of orbital invasion. COVID-19 antigen RT-PCR testing was performed on all patients to check for COVID-19 status. Contrast enhanced MRI scan of the nose, paranasal sinuses and orbit was done to evaluate disease extent.

The surgical management was decided on the disease extent as observed on clinical and radiological evaluation. Patients with disease localized to the nose and sinuses, with no involvement of the palate or orbit, underwent either endoscopic debridement via a Modified Denker's approach or a medial maxillectomy along with removal of the posterolateral wall of the maxilla. Patients with palatal involvement in the form of swelling, mucosal discoloration, or mucosal necrosis with exposure of the underlying bone, were subjected to a total maxillectomy. Patients with gross disease in the orbit on radiological evaluation with complete loss of vision and absence of perception to light on examination were subjected to orbital exenteration.

Only patients who demonstrated non-pigmented, wide, ribbon-like hyphae with no or few septations and branching at right angle confirming a diagnosis of rhinocerebral mucormycosis on $\mathrm{KOH}$ staining, fungal culture or final histopathology, were included in the study. All patients received systemic antifungal therapy viz. Amphoteric B and Posaconazole as per current treatment guidelines [4].

\section{Results}

Of the 34 patients who underwent surgery for invasive fungal sinusitis, 31 were diagnosed as mucormycosis on $\mathrm{KOH}$ staining, and were included in the present study. Of the remaining 3, two were diagnosed as belonging to Candida species, and 1 belonged to Aspergillus species, and were hence excluded from the study. Seven patients presented with intracranial extension of disease and multiple infarcts, and were not surgically fit to undergo any major procedure, and were hence not included in the present study.

Among the 31 cases included in evaluation, there were $21(67.7 \%)$ males and $10(32.3 \%)$ females with a mean age of 53.3 years (range 20-78 years). All the patients were known diabetics on treatment, with poor glycaemic control (mean HbA1c 10.2\%). Two patients were young adults suffering from Type I diabetes mellitus, while the remaining were known patients of Type II diabetes mellitus since the past 6-7 years. Twenty-three $(74.2 \%)$ were anaemic on presentation with a mean hemoglobin level of $8.4 \mathrm{gm} / \mathrm{dl}$ (range 6.4-12.8 gm/dl).

Of the 31 cases, $9(29.1 \%)$ patients were positive for COVID-19 antigen on RT-PCR testing at the time of presentation, while 17 (54.8\%) patients gave history of contracting the virus a few months prior to presentation. While none of the remaining $5(16.1 \%)$ patients gave history of COVID19 infection, HRCT Chest revealed resolving lung lesions indicating past infection with the novel coronavirus.

\section{Symptomatology}

The mean duration of symptoms was $16.8 \pm 9.8$ days. Bilateral involvement was present in $7(22.6 \%)$, while in the remaining 24 (77.4\%), only one side was affected. The most common symptoms were numbness of the cheek, headache, and visual disturbances seen in 27 (87.1\%), 26 (83.9\%) and $24(77.4 \%)$ patients, respectively (Table 1). History of loosening of teeth or palatal involvement was present in 18 $(58.1 \%)$, while nasal symptoms such as nasal obstruction or discharge was present in only $3(9.7 \%)$ patients.

\section{Nasal examination}

On diagnostic nasal endoscopy, blackening of the turbinates was seen in only 7 (22.6\%) patients and erosion of nasal floor corresponding to area of palatal necrosis was seen in $2(6.5 \%)$ patients.

Table 1 Demographic details and symptomatology

\begin{tabular}{lc}
\hline Variable & $n(\%)$ \\
\hline Males & $21(67.7)$ \\
Females & $10(32.3)$ \\
Face soft tissue involvement & \\
Normal & $9(29)$ \\
Edema & $18(58)$ \\
Cellulitis/superficial ulceration & $2(6.5)$ \\
Blackening/necrosis & $2(6.5)$ \\
Palate involvement & \\
Normal & $13(41.9)$ \\
Swelling & $2(6.5)$ \\
Mucosal darkening & $4(12.9)$ \\
Bone exposure & $7(22.6)$ \\
Eye involvement & \\
Normal & $9(29)$ \\
Preseptal cellulitis/ophthalmoplegia & $3(9.7)$ \\
Blurring of vision & $11(35.5)$ \\
Blindness & $10(32.2)$ \\
Neurological symptoms & \\
Cheek numbness & $27(87.1)$ \\
Facial palsy & $4(12.9)$ \\
\hline
\end{tabular}




\section{Face and soft tissue evaluation}

Premalar swelling and edema was seen in 18 (58\%) patients, and cellulitis with superficial ulceration was seen in $2(6.5 \%)$ patients. Complete necrosis with blackening of the overlying skin was seen in $2(6.5 \%)$ patients with history of preceding cellulitis in both (Fig. 1).

\section{Palate evaluation}

Two patients $(6.5 \%)$ had swelling of the hard palate with normal overlying mucosa. Darkening of the mucosa was seen in $4(12.9 \%)$, while mucosal necrosis with exposure of the underlying bone was seen in 7 (22.6\%) patients (Fig. 2). In 5 patients (16.1\%), although the mucosa appeared normal, there was associated loosening of teeth pointing towards underlying bone necrosis.
Fig. 1 Face soft tissue involvement. a Right malar swelling presenting with cheek numbness. $\mathbf{b}$ and $\mathbf{c}$ Cellulitis and superficial ulceration. $\mathbf{d}$ and $\mathbf{e}$ Limited and extensive soft tissue necrosis in advanced stages
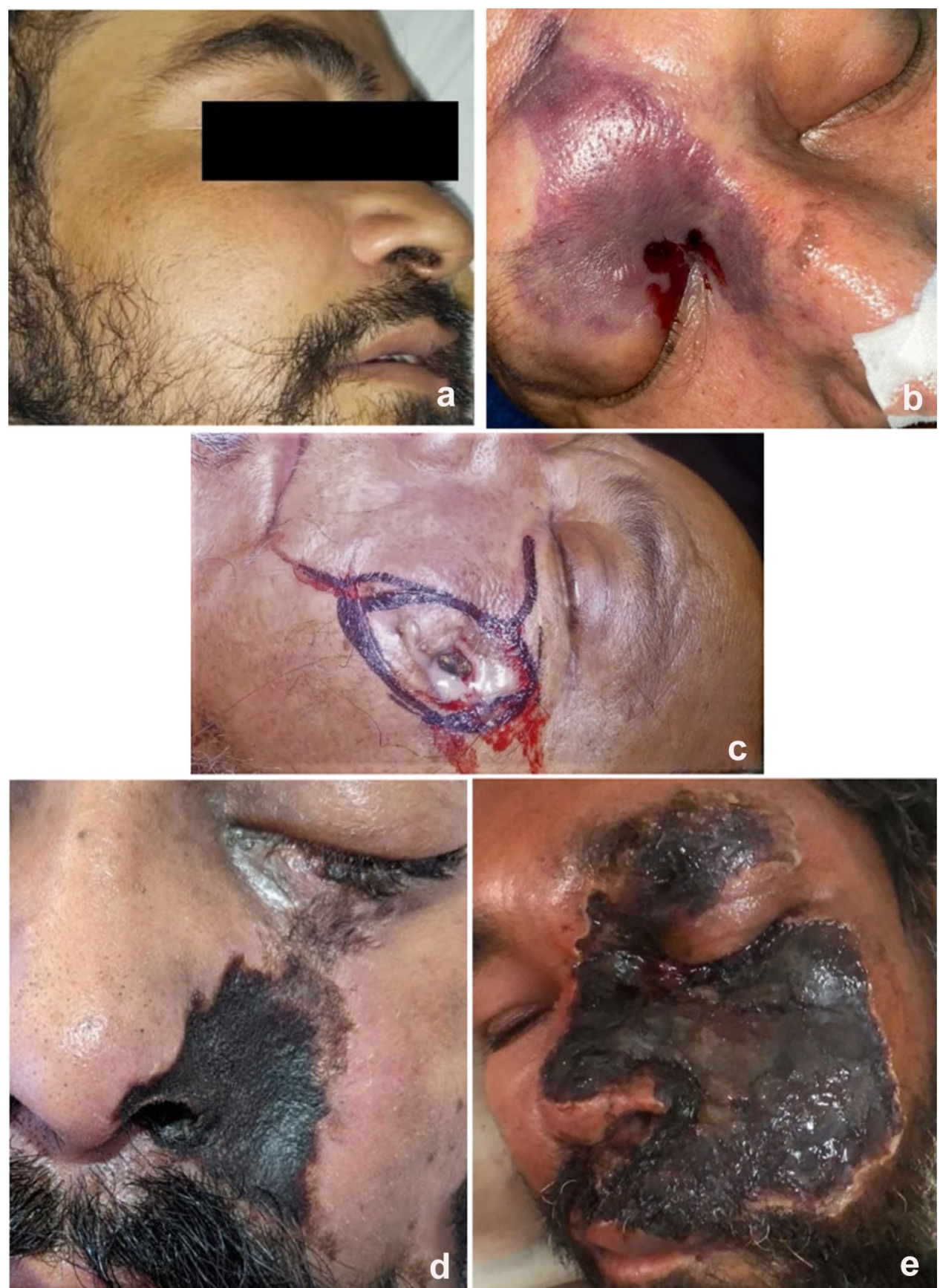
Fig. 2 Palatal involvement. a Left palatal swelling with early loosening of teeth. b Right palatal mucosal darkening with underlying bone necrosis. $\mathbf{c}$ Right palatal necrosis with bone exposure. d Midline palatal bone exposure with signs of total palatal necrosis
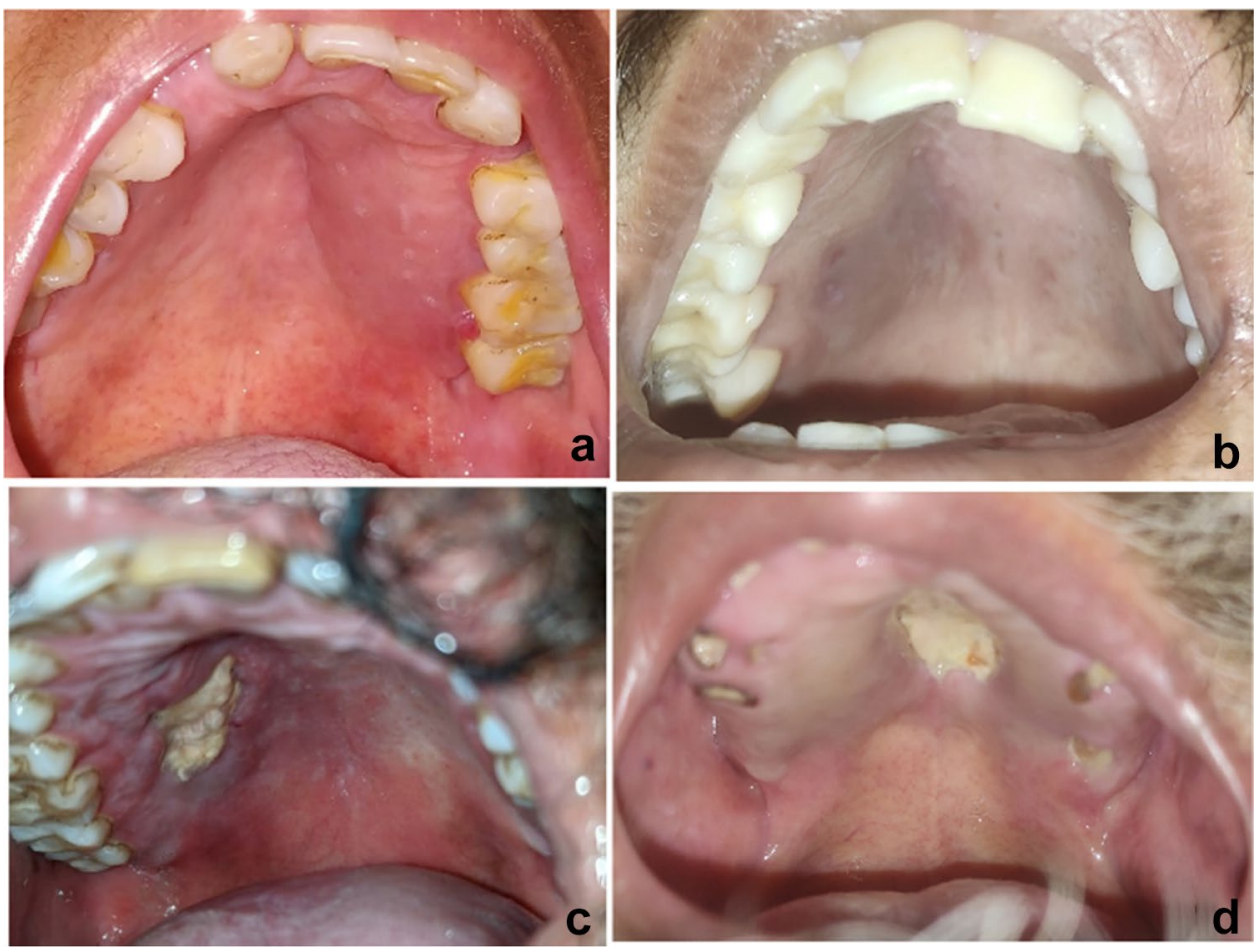

\section{Eye evaluation}

The mean duration before onset of eye symptoms was $20.3 \pm 4.6$ days. Preseptal cellulitis with or without ophthalmoplegia, but without visual disturbances was seen in $3(9.7 \%$ ) patients (Fig. 3). Blurring of vision was seen in $11(35.5 \%)$, while $10(32.2 \%)$ had complete loss of vision, of which 1 patient had loss of vision in both eyes. In a few patients, the symptoms rapidly progressed within 2-3 days, often due to the initial reluctance of patients towards extensive morbid surgeries.

\section{Neurological evaluation}

Cheek numbness was present in 27 (87.1\%) patients, all of whom had clinical involvement of the premalar soft tissue either as swelling, cellulitis or necrosis. Grade 3 infranuclear facial nerve paralysis was noted in 4 (12.9\%) patients. Of these, 2 patients had blackening of malar skin, 1 had superficial ulceration, while 1 patient had premaxillary swelling.

\section{Radiological evaluation}

Contrast enhanced MRI evaluation was done in all patients. The ethmoid sinuses were involved in all the cases, while the maxillary and sphenoid sinuses were involved in 27 (87.1\%) and $26(83.9 \%)$ cases, respectively. No case of frontal sinus involvement was seen. The cavernous sinus was involved in $14(45.2 \%)$ cases, while involvement of the pterygopalatine and/or infratemporal fossa was seen in 24 (77.4\%) cases. On correlating eye symptomatology with radiological features, of the 10 patients presenting with complete loss of vision, 2 had frank retrobulbar optic neuritis, while the remaining 8 had early features of retrobulbar neuritis with partial or
Fig. 3 Eye involvement. a Right ptosis with ophthalmoplegia suggestive of cavernous sinus thrombosis. b Advanced orbital involvement with bilateral loss of vision
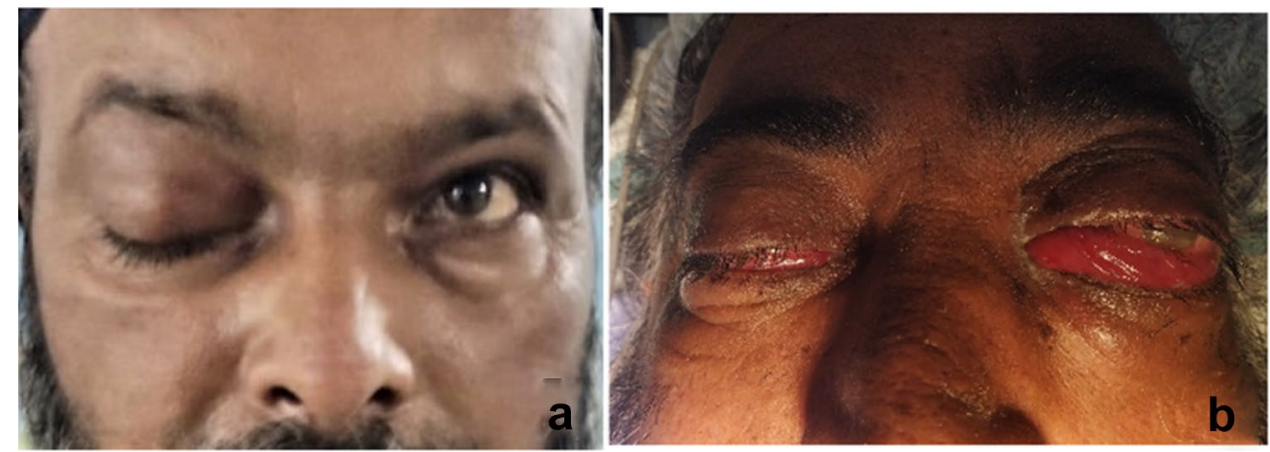
complete encasement of the optic nerve at the orbital apex. Among patients presenting with blurring of vision, soft tissue in the medial or inferior part of the anterior orbit was seen in 6 cases, retrobulbar fat stranding in 5 cases, with two of these patients having additional features suggestive of early optic neuritis.

In the patient with bilateral orbital involvement, the ipsilateral side had soft tissue involvement of the orbit with optic neuritis. This was seen to extend along the optic chiasma to involve the contralateral optic nerve at the apex, while the rest of the globe was completely uninvolved.

\section{Intraoperative details}

The aim of surgery in our patients was to attempt a complete clearance of all necrotic and devascularized bone and soft tissue, while also providing an easy access for cleaning of the resulting cavity and evaluation for any signs of recurrence during follow-up. Endoscopic clearance via Modified Denker's approach or medial maxillectomy was done in 5 (16.1\%) patients with limited involvement of only the nasal cavity and paranasal sinuses. This approach was preferred as it not only provides unhampered access to the maxillary sinus and pterygopalatine area during surgery permitting disease clearance, post-operative cleaning and surveillance. Total maxillectomy was done in $26(83.9 \%)$ patients, which included 18 patients with palatal involvement. Intraoperatively, and on preoperative imaging, all these patients had disease involving either the orbital floor, or extending along the inferior orbital fissure, necessitating total maxillectomy. In addition to these, there were 8 patients in whom the palate was apparently normal. However, on raising the cheek flap, the underlying bone was found to be yellowish, friable and devascularized, and hence a decision was taken to resect the maxilla (Fig. 4). On raising the cheek flap, the infraorbital nerve was found to be involved in 22 patients, all with cheek soft tissue involvement (Fig. 5). On tracing the infraorbital nerve proximally, the maxillary nerve from its exit at the foramen rotundum and the vidian nerve were both found to be involved. In one patient, although the maxillary and vidian nerves were found to be involved, the infraorbital nerve as well as the premaxillary soft tissue were normal, hinting at a possible proximal to distal spread of infection via the nerves.

Inspection of the pterygopalatine fossa (PPF) was done in all patients except 4 with disease limited to the nasal
Fig. 4 Intraoperative findings. a Necrosis of subcutaneous tissues (thick arrow) and exposed necrosed bone in left upper gingivobuccal sulcus (thin arrow). b Maxillary bone necrosis and intrasinus fungal deposits presenting with loosening of teeth. c and d Fungal soft tissue shadow seen behind posterolateral wall of maxilla before and after bone removal
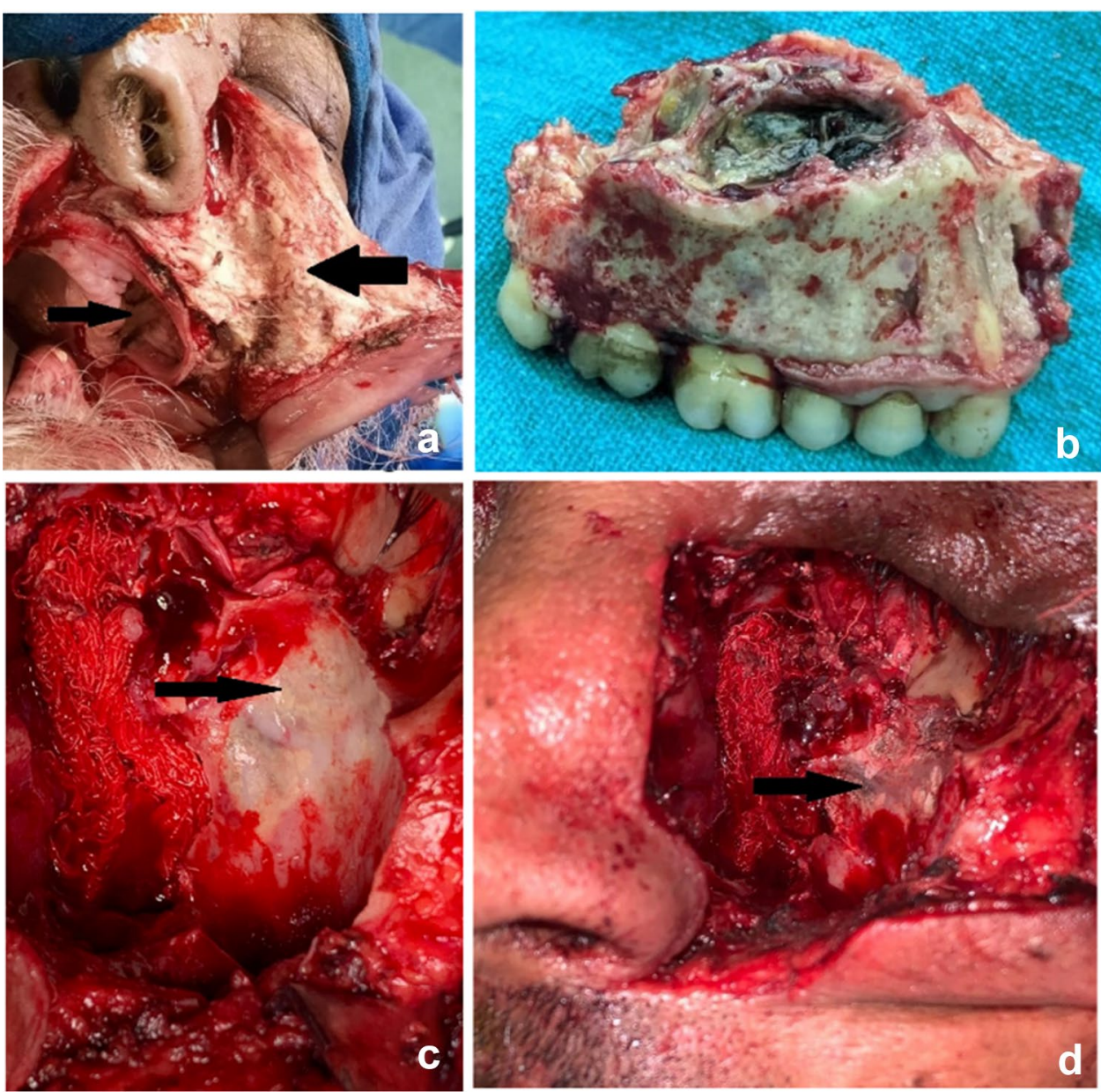
Fig. 5 Neurovascular spread. a Disease extending into orbit along ethmoidal neurovascular bundle seen during orbital exenteration. b Disease extending along infraorbital nerve with necrosed cheek soft tissue
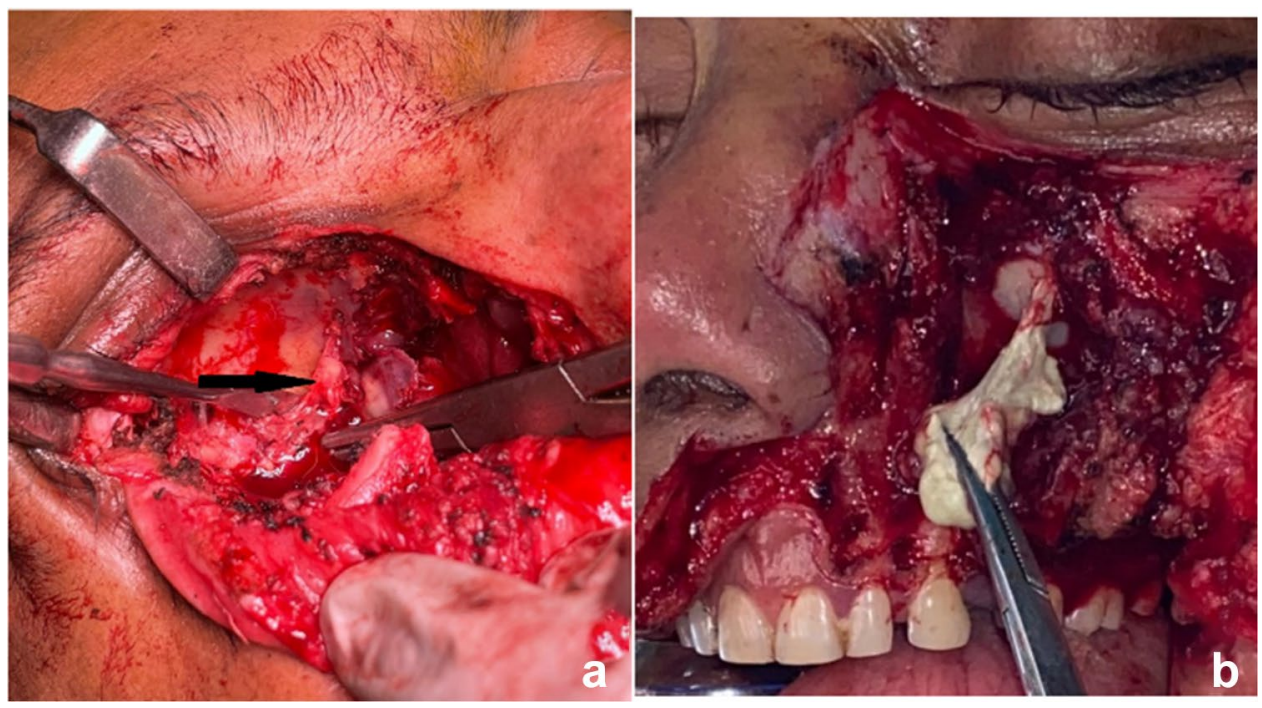

cavity and minimal involvement of the ethmoid sinus. All patients with radiological evidence of disease in the PPF (24 patients; $77.4 \%$ ) were found to harbour disease on inspection and underwent complete disease clearance, while in the 3 patients without radiological evidence of disease, the pterygopalatine fossa was normal. All 10 patients with complete loss of vision underwent orbital exenteration, included one female who underwent bilateral orbital exenteration.

Prosthetic rehabililtation was done in all patients requiring maxillectomy. Patients were counseled for microvascular reconstruction as a second-stage procedure after a symptomfree period of 6 months.

\section{Follow-up}

The mean duration of follow-up was 51.6 days (range 15-120 days). Of the 31 cases operated, there were 8 (25.8\%) deaths. The cause of death was extensive intracranial extension, sepsis, cardiac arrest, and post COVID sequelae in two patients each. Among the remaining patients, 5 (16.1\%) are discharged and are currently asymptomatic, while $18(58.1 \%)$ are undergoing treatment.

\section{Discussion}

Looking at the etiology of mucormycosis in the present study population, it was no surprise that all patients were known diabetics with poor glycaemic control. In diabetic ketoacidosis, the acidic $\mathrm{pH}$ causes the iron-protein complexes in the blood to dissociate, making the free iron available for the fungal cells [5]. This mopping up of the serum iron by the fungal cells could explain the high incidence of anaemia seen among our patients.
However, more interestingly, all patients either tested positive for COVID-19 on presentation, gave history of testing positive in the prior months, or had radiological evidence of past infection with the COVID-19 virus. This association can be explained by the fact that besides severely compromising the immunity of the host, the use of steroids in the treatment regimen of COVID-19 infection could lead to impaired macrophage function and poor glycaemic control, predisposing to opportunistic infections such as mucormycosis $[6,7]$.

Angioinvasion and hematogenous spread are hallmarks of mucormycosis. The fungal hyphae grow along the internal elastic lamina leading to intimal hyperplasia and intravascular thrombosis leading to vessel occlusion [8, 9]. A similar pathogenesis of thrombotic microangiopathy is also present in COVID-19 infection, which could probably have a synergicstic effect with mucormycosis, leading to a fulminant course with rapid dissemination of infection.

Following inhalation of the sporangiospores, the nasal cavity is the first to be affected, resulting in nasal obstruction due to thick discharge and crusts with blackening and necrosis of the turbinates and nasal septum [10, 11]. This may, however, not be evident in all patients, as can be seen in the present study, with blackened necrotic turbinates present in only 7 (22.6\%) patients. The infection then spreads to the paranasal sinuses, to the orbit causing preseptal cellulitis, ophthalmoplegia, optic neuritis and loss of vision, or to the cavernous sinus with intracranial extension. The infection may even spread inferiorly resulting in necrosis of the hard palate, or anteriorly involving the soft tissue of the cheek [12].

This, however, does not occur through direct extension, as is evident by the absence of bone erosion of the posterolateral or anterior walls of the maxilla, the lamina papyracea, or the hard palate. Following inhalation of spores, the infection 
travels via the sphenopalatine foramen to the pterygopalatine fossa. The pterygopalatine fossa then acts as the epicentre of the disease providing a pathway for the spread of infection to various sites along the various nerves and vessels in relation to it $[13,14]$. Orbital invasion may follow one of the following paths. Following infection of the maxillary nerve in the pterygopalatine fossa, the disease may spread along the infraorbital nerve to involve the inferior orbital cavity or the retrobulbar space, while in the presence of predominant ethmoidal disease, it may course along the ethmoidal vessels to predominantly involve the medial orbital cavity. In both these instances, the lamina papyracea and the orbital floor although yellowish, thinned out and friable, were structurally intact, supporting this theory. In three of our patients with a large, well pneumatised sphenoid sinus, the disease was found to preferentially spread through it to the cavernous sinus, involving the optic nerve at the orbital apex.

The infraorbital nerve is one of the commonest nerves to be affected. Once affected, the patient will complain of pain and paraesthesia of the malar region, often the first symptom of the patient $[14,15]$. In the present study, cheek numbness was present in 27 (87.1\%) patients, all with infection of the cheek soft tissues. This indicates that the nerve may act as a conduit for the spread of infection from the pterygopalatine fossa to the premaxillary soft tissues. In the earliest stages, there may just be evidence of malar edema. This then progresses to cellulitis with superficial ulceration and finally, with progressive thrombotic microangiopathy, complete blackening and necrosis of the overlying skin. Extensive necrosis of the facial muscles may also result in facial nerve paralysis, as was seen in 4 (12.9\%) of our patients.

In the palate too, stages of involvement were observed, with some patients presenting with palatal swelling with normal overlying mucosa, or with darkening of the palatal mucosa. Both these stages may be associated with loosening of teeth indicating the presence of underlying maxillary osteomyelitis. Eventually, thrombosis results in necrosis of the palatal mucosa with exposure of the underlying bone. This can also be attributed to the involvement of neurovascular structures including the maxillary artery and its terminal branches as it courses through the pterygopalatine fossa.

The pterygopalatine fossa was involved in 24 (77.4\%) cases in the present study, and is an extremely important site harbouring the mucor infection. It often acts as the epicentre of disease with dissemination of infection occurring through the various nerves and vessels coursing through this region, as well as the communicating spaces such as the inferior orbital fissure and the infratemporal fossa $[14,16]$. Moreover, the disease in this area may not be evident on initial evaluation, and may be revealed only on removing the apparently healthy posterolateral wall of the maxilla and the pterygoid plates. Fortunately, radiological evaluation with contrast enhanced MRI is an accurate tool in identifying involvement of the pterygopalatine or infratemporal fossa as can be seen from the present study. However, in view of the high incidence of involvement and rapid progression of disease, routine exploration of the pterygopalatine fossa must be performed in all cases.

The main limitations of the present study are the smaller number of patients with varied presentations and the short follow-up period. Long term analysis of the present study population and more such patients presenting to us, will shed more light on the disease biology, factors affecting outcome, and correlation of the disease with newer variants of the COVID-19 virus.

\section{Conclusion}

Mucormycosis is a rapidly progressive fungal infection with high morbidity and mortality despite surgical debridement and systemic antifungal therapy. A sudden surge in the cases of mucormycosis in the COVID-19 pandemic is probably due to a compromise in immunity along with a synergistic effect in thrombotic microangiopathy, resulting in a more fulminant course. Due to vague initial symptoms and ignorance related to disease symptomatology, patients often present in advanced stages once the infection causes palatal and facial soft tissue necrosis, or loss of vision. Spread of infection to the soft tissues of the infratemporal fossa, orbit or palate occur via neurovascular structures rather than by bone erosion. When present, infection preferentially spreads through a well pneumatized sphenoid sinus to involve the cavernous sinus and orbital apex. The pterygopalatine fossa is involved in most individuals, hidden behind apparently normal bone, and should be explored in all patients. Although considered a pathognomic feature of mucormycosis, the absence of blackened necrotic eschar on diagnostic nasal endoscopy does not preclude a diagnosis of mucormycosis. A high index of suspicion is needed in any patient of uncontrolled diabetes, especially with history of COVID-19 infection, presenting with maxillary osteomyelitis, and should prompt immediate radiological evaluation.

Author contributions PP: Study design, data acquisition, analysis, and interpretation, drafting the article and revising it critically for intellectual content. BS: Conception of the study, data acquisition and its interpretation, revising the draft critically for important intellectual content and final approval of the version to be published. SS: Study design, data acquisition, analysis, and interpretation, drafting the article and revising it critically for intellectual content. RK: Study design, data acquisition, analysis, and interpretation, drafting the article and revising it critically for intellectual content.

Funding None. 


\section{Declarations}

Conflict of interest The authors have no conflict of interest to declare.

Ethical approval Approval was obtained from the Institutional Ethics Committee, and all procedures contributing to this work comply with the ethical standards of the relevant national and institutional guidelines and with the Helsinki Declaration of 1975, as revised in 2008.

Consent to participate Appropriate consent was taken from patients.

Consent for publication Appropriate consent was taken from patients.

Availability of data and materials Medical records and data regarding all patients is available.

Code availability NA.

\section{References}

1. Skiada A, Pavleas I, Drogari-Apiranthitou M (2020) Epidemiology and diagnosis of mucormycosis: an update. J Fungi 6:265

2. Prabhu RM, Patel R (2004) Mucormycosis and entomophthoramycosis: a review of the clinical manifestations, diagnosis and treatment. Clin Microbiol Infect 10:31-47

3. Chander J, Singla N, Kaur M, Punia RS, Attri A, Alastruey-Izquierdo A, Stchigel AM, Cano-Lira JF, Guarro J (2017) Saksenaea erythrospora, an emerging mucoralean fungus causing severe necrotizing skin and soft tissue infections - a study from a tertiary care hospital in north India. Infect Dis 49:170-177

4. Cornely OA, Alastruey-Izquierdo A, Arenz D, Chen SCA, Dannaoui E, Hochhegger B et al (2019) Global guideline for the diagnosis and management of mucormycosis: an initiative of the European Confederation of Medical Mycology in cooperation with the Mycoses Study Group Education and Research Consortium. Lancet Infect Dis 19:405-421

5. Rammaert B, Lanternier F, Zahar JR, Dannaoui E, Bougnoux ME, Lecuit M, Lortholary O (2012) Healthcare-associated mucormycosis. Clin Infect Dis 54(Suppl. 1):S44-S54
6. Kontoyiannis DP, Lewis RE (2011) How I treat mucormycosis. Blood 118:1216-1224

7. Jung SH, Kim SW, Park CS, Song CE, Cho JH, Lee JH et al (2009) Rhinocerebral Mucormycosis: consideration of prognostic factors and treatment modality. Auris Nasus Larynx 36:274-279

8. Chikley A, Ben-Ami R, Kontoyiannis DP (2019) Mucormycosis of the central nervous system. J Fungi 5:59

9. Tiwari NR, Phatak S, Sharma VR, Agarwal SK (2021) COVID-19 and thrombotic microangiopathies. Thromb Res 202:191-198

10. Walsh TJ, Skiada A, Cornely OA, Roilides E, Ibrahim A, Zaoutis T, Groll A, Lortholary O, Kontoyiannis DP, Petrikkos G (2014) Development of new strategies for early diagnosis of mucormycosis from bench to bedside. Mycoses 57:2-7

11. Yohai RA, Bullock JD, Aziz AA, Markert RJ (1994) Survival factors in rhino-orbital-cerebral mucormycosis. Surv Ophthalmol 39:3-22

12. Kemper J, Kuijper EJ, Mirck PGB, Balm AJM (1993) Recovery from rhinocerebral mucormycosis in a ketoacidotic diabetic patient: a case report. J Laryngol Otol 107:233-235

13. Hussain S, Salahuddin N, Ahmad I, Salahudin I, Jooma R (1995) Rhinocerebral invasive mycosis: occurrence in immunocompetent individuals. Eur J Radiol 20:151-155

14. Hosseini SMS, Borghei P (2005) Rhinocerebral mucormycosis: pathways of spread. Eur Arch Otorhinolaryngol 262:932-938

15. Raj P, Vella EJ, Bickerton RC (1998) Successful treatment of rhinocerebral mucormycosis by a combination of aggressive surgical debridement and the use of systemic liposomal amphotericin B and local therapy with nebulized amphotericin - a case report. J Laryngol Otol 112:367-370

16. Brown RB, Lau SK (2001) A 59-year-old diabetic man with unilateral visual loss and oculomotor nerve palsy. N Engl J Med 344:286-293

Publisher's Note Springer Nature remains neutral with regard to jurisdictional claims in published maps and institutional affiliations. 